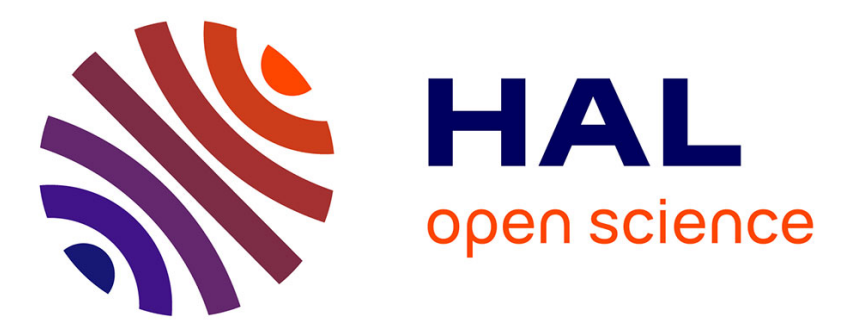

\title{
Histoire de la prise en charge des cancers broncho-pulmonaires non à petites cellules de stade précoce: de la chirurgie à la radiothérapie stéréotaxique
} Annaig Bertho, Morgane dos Santos, Agnes Francois, Fabien Milliat

\section{- To cite this version:}

Annaig Bertho, Morgane dos Santos, Agnes Francois, Fabien Milliat. Histoire de la prise en charge des cancers broncho-pulmonaires non à petites cellules de stade précoce: de la chirurgie à la radiothérapie stéréotaxique. Radioprotection, 2020, 55 (3), pp.165-172. 10.1051/radiopro/2020050 . hal-03167222

\author{
HAL Id: hal-03167222 \\ https://hal.science/hal-03167222
}

Submitted on 11 Mar 2021

HAL is a multi-disciplinary open access archive for the deposit and dissemination of scientific research documents, whether they are published or not. The documents may come from teaching and research institutions in France or abroad, or from public or private research centers.
L'archive ouverte pluridisciplinaire HAL, est destinée au dépôt et à la diffusion de documents scientifiques de niveau recherche, publiés ou non, émanant des établissements d'enseignement et de recherche français ou étrangers, des laboratoires publics ou privés. 


\title{
Histoire de la prise en charge des cancers bronchopulmonaires non à petites cellules de stade précoce: de la chirurgie à la radiothérapie stéréotaxique
}

\author{
A. Bertho ${ }^{1}$, M. Dos Santos $^{2}$, A. François ${ }^{1, *}$ et F. Milliat ${ }^{1}$ \\ ${ }^{1}$ Institut de radioprotection et de sÛreté nucléaire (IRSN), service de recherche en radiobiologie et en médecine régénérative, laboratoire \\ de radiobiologie des expositions médicales, 31 avenue de la Division Leclerc, 92260 Fontenay-aux-Roses, France. \\ ${ }^{2}$ Institut de radioprotection et de sÛreté nucléaire (IRSN), service de recherche en radiobiologie et en médecine régénérative, laboratoire \\ de radiobiologie des expositions accidentelles, 31 avenue de la Division Leclerc, 92260 Fontenay-aux-Roses, France.
}

Reçu le 26 novembre 2019 / Accepté le 21 avril 2020

\begin{abstract}
Résumé - Avant le début du XX ${ }^{\complement}$ siècle, le cancer bronchopulmonaire était une maladie rare. Aujourd'hui, c'est le quatrième cancer le plus fréquent en France et concerne, chaque année, près de 50000 patients. Si à travers l'histoire, la pierre angulaire de la prise en charge thérapeutique du cancer bronchopulmonaire reste la chirurgie, la radiothérapie en est un des piliers, notamment chez les patients à haut risque chirurgical. La radiothérapie est apparue quelques mois après la découverte des rayons X en 1896 et, rapidement, des protocoles standardisés ont été mis au point par les premiers radiobiologistes. Ces protocoles sont ceux que nous connaissons encore aujourd'hui : 2 Gy par fraction et 5 fractions par semaine sur une durée totale de 5 à 8 semaines. Si les protocoles ont peu changé en un siècle, la technique et la balistique ont connu de grandes avancées. Ces améliorations ont mené à un bouleversement profond des protocoles. Les améliorations techniques de délivrance de dose, par l'optimisation de l'imagerie, de la précision du positionnement des patients et dans la modulation de la géométrie des faisceaux ont conduit au développement de la radiothérapie en conditions stéréotaxiques ou radiothérapie stéréotaxique. Aujourd'hui, la radiothérapie stéréotaxique est utilisée pour la prise en charge des tumeurs bronchopulmonaires de stade précoce comme alternative à la chirurgie.
\end{abstract}

Mots clés : cancer pulmonaire / chirurgie / radiothérapie / radiothérapie stéréotaxique / pneumopathie radique

\begin{abstract}
Before the beginning of the 20th century, bronchopulmonary cancer was a rare disease. Today, it is the fourth most common cancer in France and affects nearly 49,000 patients each year. If throughout history, the gold standard treatment of bronchopulmonary cancer remains surgery, radiotherapy is one of the pillars, especially in patients at high surgical risk. Radiotherapy appeared a few months after the discovery of X-rays, in 1896, and quickly standardized protocols were developed by the first radiobiologists. These protocols are those we still know today: 2 Gy per fraction and 5 fractions per week over a total period of 5 to 8 weeks. If the protocols have changed little in a century, the technique and ballistics have known great advances. These improvements have led to a dramatic change in dose delivery protocols. Technical advances in dose delivery, via imaging optimization, accuracy in patients positioning and in beam geometry modulation led to the development of a new kind of radiotherapy: the radiotherapy in stereotaxic conditions or stereotaxic body radiation therapy. Today, stereotaxic body radiation therapy is used to manage early stage bronchopulmonary tumors as an alternative to surgery.
\end{abstract} Q2

\section{Introduction}

Les débuts de la prise en charge des cancers pulmonaires remontent au début du $\mathrm{XX}^{\mathrm{e}}$ siècle. En effet, avant les années

\footnotetext{
* Auteur de correspondance : agnes.francois@irsn.fr
}

1930 , le cancer bronchopulmonaire était une maladie rare, comme en témoigne Alton Ochsner (1896-1981), chirurgien : «As a junior student in 1919 at Washington University, I recall very vividly seeing a patient with cancer of the lung who was admitted to the Barnes Hospital. As is usual, the patient died. Dr Georges Dock, our eminent professor of medicine, who was also a great pathologist, insisted upon having the two senior 
classes witness the autopsy because he said the condition was so rare he thought we might never see another case as long as we lived. [...] I did not see another case until 1936, seventeen years later, when in a period of six months, I saw nine cases of cancer of the lung 》 (Ochsner, 1978). Cette augmentation du nombre de cas de cancer du poumon est liée à l'apparition de la cigarette industrielle en 1843 qui va s'imposer comme un produit de consommation courante après la Première Guerre mondiale.

Aujourd'hui, en France, le cancer bronchopulmonaire concerne près de 50000 nouveaux patients par an. Parmi eux, $85 \%$ sont des cancers bronchopulmonaires non à petites cellules (CBNPC). La pierre angulaire de leur prise en charge thérapeutique est la chirurgie, si l'état du patient le permet. Pour les patients inopérables à CBNPC de stade précoce, la radiothérapie sera recommandée. La chimiothérapie étant réservée aux stades avancés, la chirurgie et la radiothérapie sont donc les traitements les plus fréquemment utilisés.

Alors que les origines de la chirurgie remontent à la préhistoire, c'est la découverte des rayons $X$ par Whilhelm Röntgen en 1895 qui va conduire au développement de la radiothérapie. Dès leur découverte, les propriétés biologiques des rayons $\mathrm{X}$ ont ouvert de nouvelles perspectives en médecine, avec, en juillet 1896, la première utilisation des rayonnements ionisants dans le but de traiter un cancer par le médecin lyonnais Victor Despeignes (Foray, 2016a, 2016b). La radiothérapie venait de voir le jour. La radiothérapie, telle qu'on la connait aujourd'hui, normofractionnée à 2 Gy par fraction et 5 fractions par semaine sur une durée totale de 5 à 8 semaines est finalement née dans les années 1920, à l'Institut du radium (ancien nom de 1'Institut Curie) des discussions entre Claudius Regaud et Henri Coutard (Cosset et al., 2013). Quasiment un siècle plus tard, la radiothérapie est utilisée dans près de $50 \%$ des cas, seule ou en association avec d'autres thérapies. Si les installations et la prise en charge des patients ont beaucoup évolué, les protocoles, eux, n'ont que très peu changé.

\section{La chirurgie}

La première résection pulmonaire de l'histoire pour traiter une tumeur pulmonaire périphérique est réalisée en 1861 par Jules-Émile Péan, un chirurgien français (Ochsner, 1978). En 1914, Chevalier Jackson (États-Unis) utilise pour la première fois la bronchoscopie pour la résection d'une tumeur bronchopulmonaire (Modlin et al., 2014). Le 5 avril 1933, Evarts A. Graham (États-Unis) (Fig. 1) réalise la première pneumonectomie réussie, sur le Dr James Gilmore, 48 ans, pour un carcinome central sur le lobe supérieur gauche. Ce qui devait être une lobectomie fut finalement la première pneumonectomie de l'histoire à cause de l'extension du carcinome au niveau de la bronche inférieure et du lobe inférieur ainsi qu'à une scissure incomplète entre les deux lobes (Horn et Johnson, 2008). Pour une trentaine d'année, la pneumonectomie devient le traitement de référence pour le cancer du poumon, malgré un taux élevé de complications et de mortalité.

La lobectomie est alors comparée à la pneumonectomie et prend place de traitement de référence en 1962 suite à l'étude de Shimkin et al. (1962) montrant une survie équivalente et

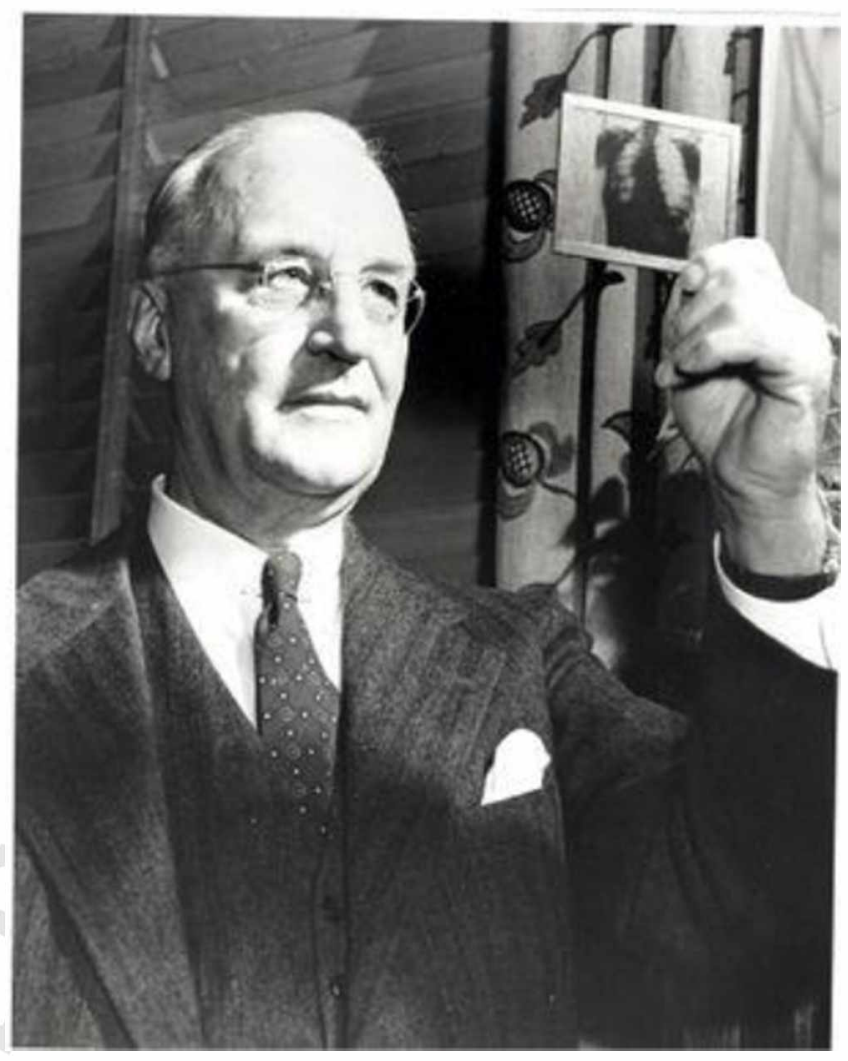

Fig. 1. Evarts Ambrose Graham, chirurgien thoracique américain, pionner des résections pulmonaires.

moins de complications postopératoires pour la lobectomie. Ces techniques, quoi qu'il en soit, nécessitent une thoracotomie, c'est-à-dire une grande incision permettant l'écartement des côtes. Dans les années 1990, la chirurgie assistée par vidéo ou thoracoscopie se développe. La thoracoscopie est une technique de chirurgie mini-invasive. Elle décrit une procédure chirurgicale avec un nombre variable d'incisions, inférieures à $8 \mathrm{~cm}$, qui permettent l'accès de l'endoscope et des instruments rigides. Cette technique dépend de la visualisation thoracoscopique et évite complètement l'écartement ou la coupe des côtes (Khaitan et D'Amico, 2018). Les deux techniques ont été comparées: la thoracotomie a été associée avec une plus grande morbidité, une moins bonne survie, ainsi qu'à des névralgies dues à l'irritation des nerfs intercostaux. La chirurgie thoracique assistée par vidéo est cependant limitée par son manque de précision, $\mathrm{dU}$ à la rigidité des instruments et à l'absence de visualisation tridimensionnelle. Elle est donc limitée à la résection de tumeurs bronchopulmonaires non à petites cellules périphériques, de stade précoce et sans adénopathies (Abbas, 2018). Les années 1990 voient également le développement des premiers robots chirurgicaux: en 1992 , le Robodoc ${ }^{\mathbb{R}}$ pour la pose de prothèses de hanche et, en 1998 , le robot DaVinci ${ }^{\mathbb{R}}$, première version standard de robot chirurgical. Ces plateformes utilisent une caméra haute résolution, tridimensionnelle et des instruments articulés capables de s'insérer dans des incisions de $8 \mathrm{~mm}$. Le chirurgien contrôle le robot via une console. Ces robots chirurgicaux permettent des actes précis, ce qui résout les limitations de la chirurgie thoracique vidéo assistée. La 
chirurgie thoracique robotisée est aujourd'hui utilisée en routine pour tous les cas de CBNPC (tumeurs périphériques ou centrales, pneumonectomic, bronchoplastie). Comparée à la thoracotomie, elle diminue la mortalité, les complications périopératoires et diminue la durée d'hospitalisation. Comparée à la chirurgie thoracique assistée par vidéo, elle permet de diminuer l'utilisation des narcotiques et permet un retour aux activités normales plus rapide. En matière d'efficacité, la lobectomie robotisée présente de bons résultats. Avec un suivi médian de 27 mois, la survie globale à 5 ans est de $80 \%$. La survie à 5 ans est comprise entre $88 \%$ et $91 \%$ pour les stades I (Abbas, 2018).

\section{La radiothérapie normofractionnée : des débuts à aujourd'hui}

La radiothérapie est un traitement locorégional des cancers. Elle utilise des rayonnements ionisants pour stopper le développement et tuer les cellules cancéreuses. Peu après leur découverte, les rayonnements, rendant visible l'intérieur du corps, sont utilisés à des fins diagnostiques puis les premiers appareils de radiographie sont développés rapidement. En juillet 1896,5 mois à peine après la découverte des rayons $\mathrm{X}$, a lieu la première radiothérapie anticancéreuse de l'histoire. Victor Despeignes, médecin lyonnais, traite un patient pour ce qu'il pense être un cancer de l'estomac (Despeignes, 1896; Foray, 2016b). Il a irradié son patient, deux fois par jour (tôt le matin et tard le soir) grâce à un tube à rayons $X$, pendant 8 jours et lui a administré un traitement accessoire. Du 4 au 12 juillet, Despeignes a noté une diminution de la douleur, jusqu'à se passer des narcotiques ainsi qu'une diminution sensible de la taille de la tumeur. Cependant, son patient décède le 24 juillet 1896 (Fig. 2).

Le premier traitement réussi par radiothérapie est attribué à Eduard Schiff, un médecin allemand, qui traita un lupus érythémateux en 1897. En décembre 1898, Pierre et Marie Curie découvrent le radium. Deux ans plus tard, les effets du radium sur la peau sont décrits par Otto Walkhof et Friedrich Giesel. Ces travaux seront confirmés par Henri Becquerel et Pierre Curie en 1901 avec leur note «L'action physiologique des rayons du radium» (Comptes rendus de l'Académie des sciences, tome 132, 3 juin 1901). En 1901, la curiethérapie voit le jour : elle consiste à mettre en contact direct une source de radium et la tumeur. Quelques années plus tard, les bases de la radiobiologie vont être établies, fondant également les bases de la radioprotection (Bourguignon et al., 2017). D'abord par Jean Bergonié (radiologiste) et Louis Tribondeau (histologiste), avec leur loi, énoncée en 1906: «Les rayons X agissent avec d'autant plus d'intensité sur les cellules que l'activité reproductrice de ces cellules est plus grande, que leur devenir karyocinétique est plus long, que leur morphologie et leurs fonctions sont moins définitivement fixées 》 (Comptes rendus de l'Académie des sciences du 10 décembre 1906). La même année, Claudius Regaud, histologiste, met en évidence la radiosensibilité des spermatogonies souches dont il se servira pour réfuter la loi de Bergonié et Tribondeau (Regaud et Blanc, 1906). Il montre également qu'une dose unique de rayons $X$, suffisante pour déclencher des lésions graves à la peau et la stérilisation des testicules chez le lapin, lorsqu'elle est fractionnée sur plusieurs jours, stérilise le lapin mais n'entraîne

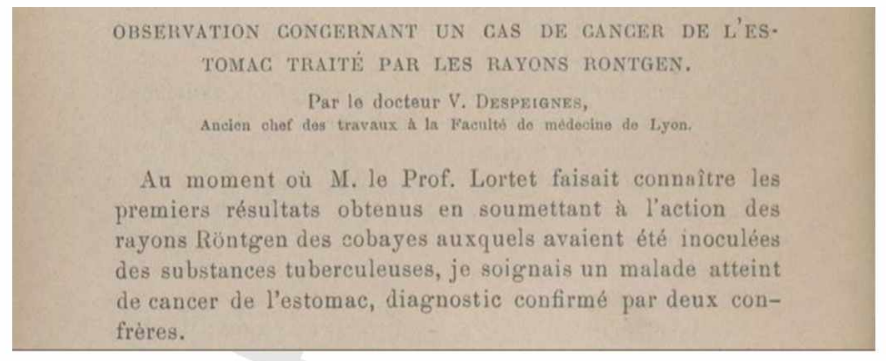

Fig. 2. Article de Victor Despeignes publié dans le Lyon Médical le 26 juillet 1896 (source: Gallica.bnf.fr / Bibliothèque nationale de France.

pas de lésion cutanée. Ces observations seront la base du principe de fractionnement en radiothérapie. C'est Henri Coutard qui appliquera les principes de Regaud à la clinique : Coutard met au point « la méthode Coutard» ou « méthode de fractionnement prolongé», consistant à administrer une forte dose de rayons $\mathrm{X}$ en fractions de faibles doses journalières sur une certaine période de temps. En 1934, Coutard publie un article: «Principles of $X$-ray therapy of malignant diseases 》, dans lequel il met 1'accent sur 1'effet du fractionnement de la dose sur le tissu conjonctif ainsi que sur la radiosensibilité de la tumeur selon différents types de cancers (Coutard, 1934). Aujourd'hui, ce principe de fractionnement de la dose est toujours appliqué. Les débuts de la radiothérapie se sont fait avec des tubes à rayons de $\mathrm{X}$ de bas voltage $(<150 \mathrm{kV})$ puis à des voltages un peu plus élevés $(200 / 500 \mathrm{kV})$ dont les rayons étaient peu pénétrants. Il était donc difficile de traiter des tumeurs profondes. Avec la découverte, en 1934, de la radioactivité artificielle par Irène et Frédéric Joliot-Curie, la production de radio-isotopes artificiels pour la radiothérapie est possible. Les premières sources de cobalt 60 ont pu être produites à partir de 1941 grâce à l'équipe du physicien Glenn Seaborg au Lawrence Berkeley National Laboratory (ÉtatsUnis). Les sources de cobalt 60 remplacent le radium, plus rare et plus cher. Les rayonnements gamma du cobalt étant plus pénétrants, les bombes à cobalt permettent le traitement de tumeurs plus profondes. Puis des appareils dédiés sont développés dans les années 1950: en 1951, le premier traitement à la télécobalthérapie est réalisé au Canada. Ce type d'appareil sera utilisé jusque dans les années 2010 en France. L'un des tournants majeurs dans l'histoire de la radiothérapie est l'apparition des accélérateurs linéaires de particules médicaux ou LINAC médicaux. Les LINAC médicaux génèrent des rayons $\mathrm{X}$ de haute énergie grâce à un faisceau d'électrons interagissant avec une cible ayant un numéro atomique élevé (tungstène, par exemple). Le premier LINAC médical est installé à Londres en 1952, le premier patient sera traité l'année suivante. Les LINAC sont les installations les plus utilisées pour la radiothérapie externe. Aujourd'hui, quand on parle de radiothérapie conventionnelle, normofractionnée à 2 Gy par fraction, 5 jours par semaine sur 5 à 8 semaines, ce terme regroupe, en fait, la radiothérapie conformationnelle 3D (3D-CRT) et la radiothérapie conformationnelle à modulation d'intensité (RCMI), toutes deux utilisées pour le traitement du CBNPC. La 3D-CRT est la technique la plus fréquemment utilisée. Elle fait correspondre au maximum le volume irradié avec le volume tumoral afin de 


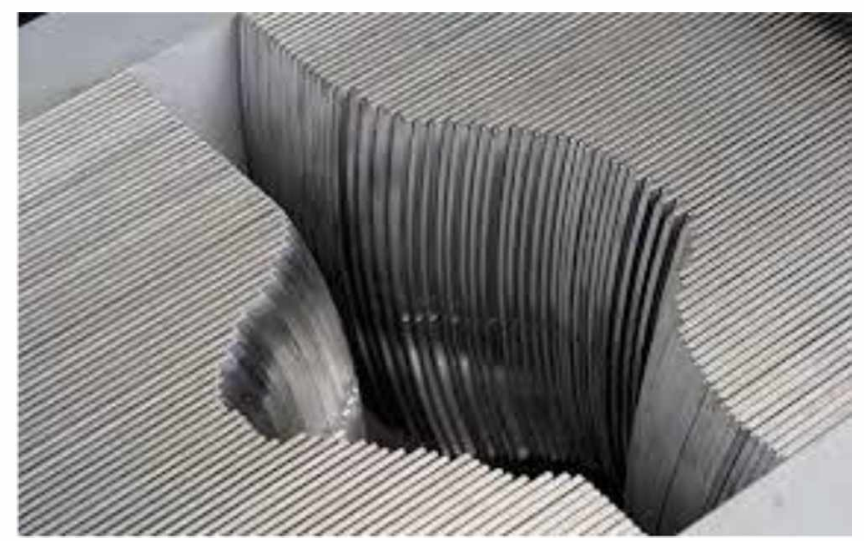

Fig. 3. Collimateur multi-lames utilisé pour moduler l'intensité du faisceau et de conformer la géométrie du faisceau sur la morphologie tumorale. délivrer une dose efficace à la tumeur tout en limitant collimateur multi-lames du LINAC (Durdux et al., 2018). La 3D-CRT fait partie des traitements curatifs du thorax.

La RCMI est une technique innovante, de plus en plus utilisée pour le thorax, notamment pour les tumeurs proches de la moelle épinière ou du cœur. Cette technique permet d'avoir un fort gradient de dose entre le volume cible clinique (CTV pour clinical target volume) et le volume de tissus à épargner. La délinéation doit de ce fait être très précise et la dosimétrie est complexe. La précision de la position du patient est donc essentielle et est vérifiée par cone beam computed tomography (CBCT). La RCMI nécessite un LINAC équipé d'un collimateur multi-lames mais également d'un logiciel de planification de traitement adapté (Fig. 3). Le flux de photons est modulé dans son intensité par le mouvement dynamique des lames du collimateur. Cette technique de modulation d'intensité permet d'irradier des volumes concaves, obtenir une résolution spatiale des faisceaux comme des angles étroits ou encore délivrer des doses inhomogènes sur le volume tumoral (De Neve et al., 1999).

Certaines variantes de la RCMI utilisent des faisceaux rotationnels: par exemple, la radiothérapie avec modulation d'intensité volumétrique par arc-thérapie (VMAT). Dans ce cas, le bras du LINAC décrit un arc de cercle. Une autre variante de la RCMI est la tomothérapie où la RCMI est en fait administrée sur un appareil dédié, le Tomotherapy ${ }^{\circledR}$ : un accélérateur miniaturisé est associé à un scanner. Cette fois, en plus de l'arc décrit par le bras de l'accélérateur, la table de traitement a un mouvement longitudinal. De cette façon, le traitement est administré de façon hélicoïdale (Chan et al., 2014). La RCMI confère un avantage dosimétrique par rapport à la 3D-CRT : le volume pulmonaire recevant 20 Gy diminue $\left(V_{20 G y}\right.$, paramètre important à prendre en compte pour limiter les séquelles du traitement) (Lefebvre et al., 2014). Yom et al. (2007) obtiennent des $V_{20 G y}$ pulmonaires de $35 \%$ avec la RCMI et de $38 \%$ avec la 3D-CRT. Liao et al. (2010) confirment ces données avec des $\mathrm{V}_{20 \mathrm{~Gy}}$ pulmonaires de $34 \%$ et $37 \%$, respectivement. Ces deux études montrent également un volume recevant $5 \mathrm{~Gy}\left(\mathrm{~V}_{5 \mathrm{~Gy}}\right)$, de 63 à $64 \%$ pour la $\mathrm{RCMI}$ alors qu'il est de $57 \%$ pour la $3 \mathrm{D}$-CRT, le volume de tissus sains l'exposition des tissus sains. Ceci est possible grâce au irradié à des doses plus faibles est donc plus important La survie était meilleure en RCMI qu'en 3D-CRT dans l'étude de Liao et al. (2010). En termes de toxicité, moins de pneumopathies radiques à 12 mois sont rapportées après RCMI que 3D-CRT. Une étude de Chun et al. (2015) montre que la RCMI est associée avec un risque moindre de pneumopathie radique. Dans une étude de Koshy et al. (2017), la RCMI est associée à une meilleure survie que la 3D-CRT. Ces dernières années, les progrès de l'imagerie et de la radiothérapie ont permis l'émergence de la radiothérapie guidée par l'image (IGRT): le LINAC est associé à un dispositif d'imagerie. L'IGRT permet d'acquérir des images anatomiques du patient, en salle, en position de traitement et permet donc de visualiser les changements survenant pendant le traitement: la dosimétrie est ainsi adaptée à la régression tumorale par exemple. Ceci permet de mieux délivrer la dose à la tumeur et de mieux protéger les organes à risque en limitant les marges sur le CTV (Durdux et al., 2018; Lefebvre et al., 2014). Une autre façon de mieux délivrer la dose au CTV tout en protégeant les organes à risque est l'asservissement respiratoire durant le traitement. Cette technique permet de prendre en compte ou de diminuer les mouvements respiratoires faisant bouger la tumeur. Quatre techniques sont actuellement disponibles : la respiration superficielle forcée où la compression abdominale diminue l'amplitude des mouvements respiratoires, le «gating» où l'irradiation a lieu sur un temps de la respiration déterminé à l'avance, le «tracking 》 où la tumeur est localisée et suivie par le faisceau d'irradiation et la respiration bloquée où le patient retient sa respiration au moment du tir, grâce à un spiromètre et des lunettes permettant de visualiser sa courbe respiratoire (Lefebvre et al., 2014) (Fig. 4).

La chirurgie et la radiothérapie restent aujourd'hui les deux piliers de la prise en charge thérapeutique des tumeurs pulmonaires. Les techniques 3D-CRT et IMRT sont largement utilisées, avec une part croissante de l'IMRT. Dans les cas de tumeurs bronchopulmonaires de stade précoce, la résection chirurgicale reste le traitement de choix offrant un bon contrôle tumoral. En revanche, pour les patients à haut risque chirurgical, la radiothérapie conventionnelle n'assure qu'un contrôle tumoral limité. Depuis quelques années, les progrès technologiques ont permis de proposer à ces patients inopérables une nouvelle technique de radiothérapie permettant un hypofractionnement sévère et assurant un contrôle tumoral comparable à la chirurgie avec peu de différences quant aux risques associés: la radiothérapie en conditions stéréotaxiques (Hamaji, 2019; Hamaji et al., 2019; Yaprak et al., 2019).

\section{La radiothérapie en conditions stéréotaxiques}

Historiquement, la stéréotaxie est une méthode de localisation des structures cérébrales via un cadre de référence externe en trois dimensions lors d'une procédure chirurgicale mini-invasive. Cette technique a été développée, chez l'animal, par Horsley et Clarke (Royaume-Uni) et publiée en 1908 (Solberg et al., 2012). Le premier cadre de stéréotaxie utilisant un système de coordonnées cartésien a été construit en 1906. Clarke s'en servira notamment pour créer des atlas d'anatomie cérébrale du singe et du chat. L'application 

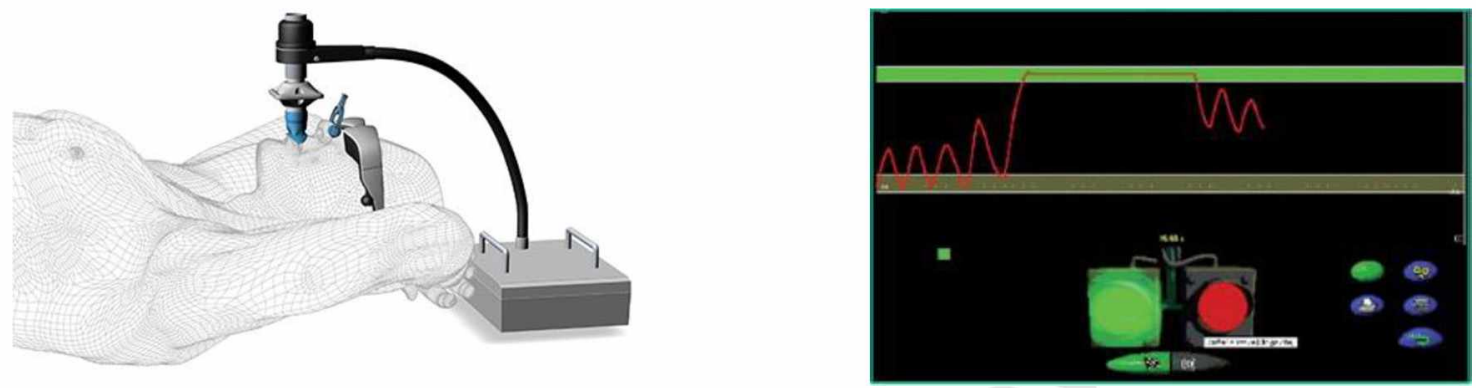

Fig. 4. Dispositif d'asservissement respiratoire lors de radiothérapie pulmonaire : la respiration bloquée. La respiration du patient est bloquée pendant l'acquisition de l'image prétraitement (à gauche). Le déclenchement du faisceau se fait à un niveau respiratoire défini (bande verte, à droite).
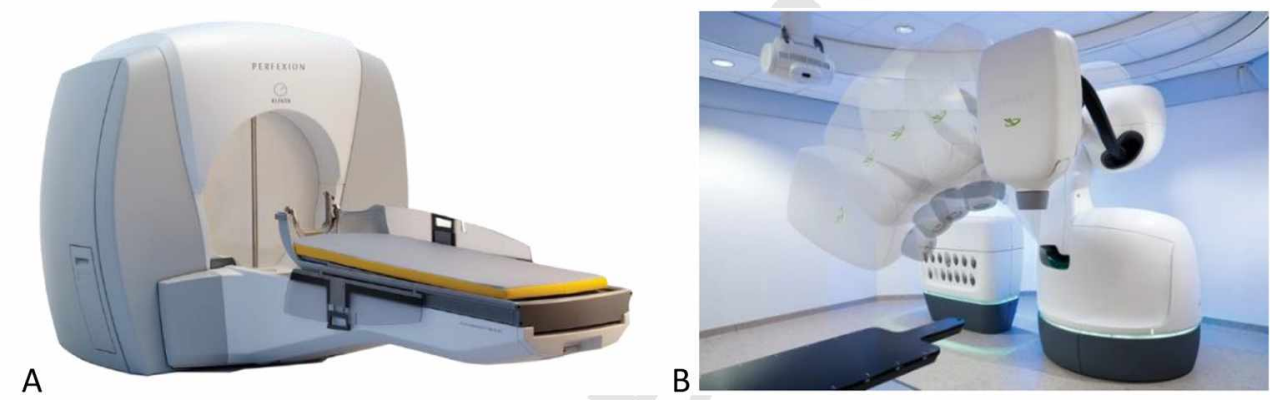

Fig. 5. Appareils de radiothérapie stéréotaxique. A. Le Gamma Knife Perfexion sorti en 2006 (source: Elekta, gammaknife.com). B. Le CyberKnife mis au point en 1987 (source: Cyberknife.com).

clinique de la stéréotaxie date de 1947. Spiegel et Wycis (ÉtatsUnis) ont mis au point un cadre de stéréotaxie, basé sur un système de coordonnées cartésiennes, similaire à celui d'Horsley et Clarke. Le cadre était fixé sur la tête du patient grâce à un plâtre amovible, rendant ainsi l'appareil adapté à la chirurgie et à l'imagerie. La stéréotaxie s'est alors développée et des améliorations y ont été apportées. Lars Leksell (Suède) mit au point un cadre de stéréotaxie basé sur un système de coordonnées polaires (angle, profondeur et position antéropostérieure). Plus tard, avec l'aide de Jenberg (Suède), il rend son cadre compatible avec la tomodensitométrie. D'autres participèrent au développement de la stéréotaxie. Le cadre de Jean Talairach (France) fut, par exemple, le premier cadre à être utilisé, en 1982, lors d'une procédure de radiochirurgie associée à un LINAC. Le développement de la radiochirurgie est d'ailleurs dÛ à John Lawrence et Cornelius Tobias (ÉtatsUnis): dès la fin des années 1940, ils conceptualisent l'utilisation de faisceaux de particules chargées pour modifier ou altérer des structures cérébrales. Puis Leksell leur suggère la méthode stéréotaxique pour guider ces faisceaux de particules. Les premières radiochirurgies ont été administrées par Leksell en 1953 et par Larsson (Suède) en 1955. La radiochirurgie à faisceaux de particules est étudiée dans différents laboratoires de physique d'Uppsala en Suède, Berkeley et Harvard aux États-Unis et Cambridge en Angleterre grâce au synchrocyclotron de Berkeley ou aux différentes installations à faisceaux de protons. En Suède, à Berkeley et à Cambridge, 1'accès aux installations était réservé à la recherche physique, l'accès pour la recherche biologique et clinique était difficile. Lars Leksell et différentes équipes de recherche suédoises se sont alors réunies pour concevoir un appareil de radiochirurgie adapté à l'hôpital : le Gamma Knife I vit le jour en 1967 à l'hôpital de Sofiahemmet à Stockholm (Solberg et al., 2012). Il est composé de 167 sources de cobalt 60 distribuées dans un secteur sphérique de $70^{\circ}$ de latitude et $160^{\circ}$ de longitude. Le collimateur fournit un faisceau de section transverse de $2,5 \times 7,5 \mathrm{~mm}$ avec une pénombre de $0,5 \mathrm{~mm}$ au centre du faisceau. Dès décembre 1967, les premiers patients sont traités, en une fraction, pour des malformations artérioveineuses intracrâniennes, des tumeurs cérébrales bénignes et malignes et des désordres fonctionnels. La décroissance des sources de cobalt 60 du Gamma Knife I conduisit à une révision de l'appareil. Le Gamma Knife II fut installé en 1975 à l'hôpital de Karolinska. Leksell suggère que cette machine pourrait être utilisée pour des traitements fractionnés: l'idée de la radiothérapie stéréotaxique est née. En 1972, Leksell fonde la société Elekta qui commercialise le Gamma Knife. Le premier Gamma Knife commercial a été installé à l'université de Pittsburg en 1987. En 2007, Elekta sort le Perfexion ${ }^{\text {TM }}$ gamma unit (Fig. 5), équipé de 192 sources de cobalt arrangées de façon conique, dans huit secteurs différents, pouvant changer de collimation: 4, 8 ou $16 \mathrm{~mm}$ de diamètre. En 2008, 500000 patients avaient été traités à travers le monde grâce aux différents modèles de Gamma Knife.

En 1982, le neurochirurgien argentin Osvaldo Betti et l'ingénieur Victor Derechinsky modifient un LINAC médical pour l'adapter à la radiochirurgie. Beaucoup d'équipes à travers le monde ont participé au développement de la radiochirurgie associée au LINAC tout au long des années 1980. Le premier LINAC pour la radiochirurgie est commercialisé à la fin des 
années 1980 par Philips Medical : le SRS 200. Il est équipé d'un bras isocentrique et de collimateurs circulaires allant de 10 à $32 \mathrm{~mm}$ et est associé à système de planification de traitement basé sur la tomodensitométrie. En 1992, le premier collimateur micromulti-lames est mis au point pour la radiochirurgie. Les années 1990 voient également l'apparition de machines dédiées pour la radiochirurgie associée au LINAC. En 1989, John Adler, neurochirurgien, conceptualise une machine de radiochirurgie constituée d'un LINAC compact robotisé, sans cadre stéréotaxique, en utilisant la superposition de deux images radiographiques pour la localisation. Le CyberKnife recevra son autorisation d'utilisation en 2001 (Fig. 5). Aujourd'hui, le CyberKnife est une machine utilisée en routine pour les traitements en conditions stéréotaxiques, intracrâniens et extracrâniens. D'autres LINAC dédiés à la radiochirurgie ont été développés: 1e 600SR (Varian), le C-arm LINAC (Mitsubishi), le Novalis. Face au succès et à l'efficacité de la radiochirurgie crâniale, son application à des tumeurs extracrâniennes pour des traitements à forte dose par fraction est évaluée dans les années 1990. La principale limite de cette application extracrânienne est la contention du patient. Contrairement aux localisations intracrâniennes, les localisations corporelles sont sujettes aux mouvements internes des organes et aux mouvements externes du patient. Dans un premier temps, des cadres corporels de stéréotaxie ont été développés par Lax et l'équipe de l'hôpital de Karolinska en Suède : ils sont associés à un dispositif de compression du thorax et de l'abdomen pour limiter les mouvements respiratoires. Le cadre était conçu pour être utilisé avec un scanner grâce à des repères radio-opaques intégrés dans le cadre de stéréotaxie. En 2003,

Q3 Yenice et al. décrivent un système de cadre stéréotaxique corporel combiné à l'image scanner quotidienne pour le repositionnement du patient. Shiu et al., eux, proposent l'installation d'un scanner sur des rails dans la salle de traitement. Aujourd'hui, la technologie CBCT est largement utilisée pour la localisation du PTV (pour planning target volume, correspondant au volume cible planifié) mais également pour l'évaluation des variations anatomiques intrafractions (mouvements respiratoires et cardiaques) et interfractions (perte de poids des patients, modification du volume tumoral). Le développement de la radiothérapie stéréotaxique extracrânienne pulmonaire est également supporté par le développement de méthodes d'asservissement respiratoire telles que la compression abdominale, le «gating» ou 1e «tracking» mentionnés précédemment. De nouvelles techniques de gestion des mouvements du volume cible se développent: l'adaptation en temps réel de la forme et de la position des faisceaux grâce aux mouvements des lames du collimateur multi-lames permet de suivre les mouvements de la tumeur, c'est le «tracking multileaf collimator» (tracking MLC). Les tables de traitement robotisées peuvent également servir à compenser les mouvements de la cible. Depuis quelques années, se développe un système de «gating 》 associé à l'imagerie par résonance (IRM) : en effet, l'IRM permet d'obtenir un meilleur contraste au niveau des tissus mous comme le poumon (Dieterich et al., 2018). L'IRM associée à la radiothérapie stéréotaxique pulmonaire pourrait permettre d'améliorer la délimitation des organes à risque, le positionnement du patient, le contrôle des mouvements internes et les adaptations (système MRIDIAN Linac, Viewray, Cleveland, États-Unis). Ceci pourrait améliorer la qualité de la radiothérapie stéréotaxique pulmonaire, notamment en améliorant sa précision, permettant de délivrer des doses plus fortes (Menten et al., 2017).

Aujourd'hui, la radiothérapie stéréotaxique extracrânienne est définie comme «une méthode de radiothérapie externe utilisée pour délivrer de fortes doses de rayonnements à une cible extracrânienne, dans le corps, utilisant soit une dose unique, soit un petit nombre de fractions" (Franks et al., 2015). Pour la radiothérapie stéréotaxique, les doses par fraction sont dites ablatives (de 6 à $20 \mathrm{~Gy}$ ): elles permettent l'ablation de la tumeur et des tissus situés dans le PTV grâce à la mort cellulaire et aux dommages vasculaires induits par de telles doses. Ces fortes doses peuvent être délivrées au PTV grâce à la précision millimétrique de la radiothérapie stéréotaxique ainsi qu'à la forte décroissance de dose en dehors du PTV: elle permet d'épargner au maximum les tissus sains. Actuellement, la radiothérapie stéréotaxique est indiquée pour des localisations intracrâniennes (métastases cérébrales, méningiomes, neurinomes de l'acoustique, tumeurs pituitaires, malformations artérioveineuses, névralgie du nerf trijumeau) et des localisations extracrâniennes (lésions médullaires, oligométastases pulmonaires, osseuses ou hépatiques, cancer de la prostate, carcinomes hépatocellulaires inopérables, CBNPC de stade I inopérables, ré-irradiations des voies aérodigestives supérieures ou récidives pelviennes).

Concernant le poumon actuellement, la radiothérapie stéréotaxique est indiquée en cas de CBNPC de stade précoce, sans invasion ganglionnaire ni métastases, avec une taille tumorale inférieure à $7 \mathrm{~cm}(\mathrm{~T} 1 \mathrm{ou} \mathrm{T} 2, \mathrm{~N} 0, \mathrm{M} 0)$. Globalement, la radiothérapie stéréotaxique est une excellente alternative thérapeutique pour les patients à haut risque chirurgical ou refusant la chirurgie d'exérèse, qui reste la première intention. Le taux de contrôle local est bon, allant de 86 à $98 \%$ à 3 ans (Lefebvre et al., 2014). Le taux de survie spécifique est de 73 à $82 \%$ et la survie globale de 43 à $57 \%$ (Menoux et al., 2018). En matière d'efficacité, quand la radiothérapie stéréotaxique pulmonaire est comparée à la 3D-CRT, l'étude de Widder et al. (2011) conclut que la survie globale et le contrôle local sont meilleurs après radiothérapie stéréotaxique : survie globale à $48 \%$ pour la $3 \mathrm{D}$-CRT contre $72 \%$ pour la radiothérapie stéréotaxique, et un contrôle tumoral de $78 \%$ pour la $3 \mathrm{D}$-CRT et $95 \%$ pour la radiothérapie stéréotaxique. La qualité de vie globale, la fonction pulmonaire et les dyspnées étaient stables après radiothérapie stéréotaxique (Widder et al., 2011). L'essai clinique randomisé de phase II, Stereotactic Precision And Conventional radiotherapy Evaluation (SPACE), compare également l'efficacité de la radiothérapie stéréotaxique et de la 3D-CRT. Les patients traités par 3D-CRT ont eu une aggravation de leur dyspnée, douleurs thoraciques et toux. Les auteurs n'ont pas trouvé de différence de survie globale entre les deux traitements. Le contrôle local est meilleur avec la radiothérapie stéréotaxique et les patients ont une meilleure qualité de vie liée à la santé ainsi que moins de toxicité radioinduite que les patients traités par 3D-CRT (Nyman et al., 2016). Les derniers résultats publiés concernant la comparaison de la radiothérapie stéréotaxique pulmonaire versus la radiothérapie conventionnelle sont ceux de l'essai clinique randomisé de phase II CHISEL pour les CBNPC de stade I. Ils concluent que le traitement par radiothérapie stéréotaxique procure un meilleur contrôle local de la maladie sans augmenter le risque de toxicité (Ball et al., 2019). L'essai clinique de phase 2 randomisé RTOG0915 compare deux types 
de traitement par radiothérapie stéréotaxique pour des CBNPC de stade I : 34 Gy en une fraction ou 48 Gy en quatre fractions. Ces deux protocoles n'ont pas entrainé de toxicité particulière et le taux de contrôle local à 5 ans est similaire. L'efficacité des deux traitements est également similaire avec une survie à 4 ans comparable (Videtic et al., 2019). Une autre étude de Koshy et al. (2015) met en évidence que des doses plus importantes, avec une biological effective dose (BED) supérieure à $150 \mathrm{~Gy}$ sont plus efficaces pour les patients atteints de CBNPC de stade II : les patients ont une meilleure survie que ceux traités avec une BED inférieure à 150 Gy. Toutes ces études sont en accord avec le fait que la radiothérapie stéréotaxique est également plus pratique pour les patients et les praticiens: le nombre de séances de traitement est généralement réduit de 35 à trois. De plus, la radiothérapie stéréotaxique diminue le cô̂t du traitement (Lanni et al., 2011). Toutes ces données font de la radiothérapie stéréotaxique pulmonaire le traitement de référence pour les patients inopérables ou refusant la chirurgie atteints de CBNPC de stade précoce.

\section{Conclusion}

La radiothérapie s'est développée dans les quelques années qui ont suivi la découverte des RX. Depuis un peu plus de 120 ans maintenant, la radiothérapie a connu des avancées majeures concernant les appareils délivrant la dose mais également dans les techniques d'imagerie et de positionnement des patients prenant en compte les mouvements de la tumeur et des tissus sains. Ce sont ces progrès balistiques qui ont permis d'améliorer sans cesse à la fois la qualité du contrôle tumoral et la protection des tissus sains. Les progrès balistiques permettant d'irradier de tout petits volumes ont également permis, ces 10 dernières années, de modifier drastiquement les schémas de fractionnement et d'appliquer des protocoles de radiothérapie avec des doses par fraction dites ablatives, de 3 à 10 fois plus élevées que lors d'un fractionnement classique. Globalement, si la radiobiologie est née quasiment en même temps que la radiothérapie, les nouvelles techniques et les nouveaux schémas de délivrance de dose, en faisant varier les volumes exposés et les doses délivrées, changent complètement la radiobiologie des tissus irradiés, qu'ils soient tumoraux ou sains. Comme souvent, les applications cliniques précèdent les connaissances radiobiologiques. Ce qui pose alors la question du développement de modèles précliniques, cliniquement pertinents du point de vue de la délivrance de la dose, permettant d'étudier les réactions des tissus sains et tumoraux aux fortes doses par fraction.

\section{Références}

Abbas E. 2018. Surgical management of lung cancer: history, evolution, and modern advances. Curr. Oncol. Rep. 20(12): 98.

Ball D, et al. 2019. Stereotactic ablative radiotherapy versus standard radiotherapy in stage 1 non-small-cell lung cancer (TROG 09.02 CHISEL): a phase 3, open-label, randomised controlled trial. Lancet Oncol. 20(4): 494-503.

Bourguignon M, Bérard P, Bertho J, Farah J, Mercat C. 2017. What's next in radioprotection? Radioprotection 52: 21-28.
Chan C, et al. 2014. Intensity-modulated radiotherapy for lung cancer: current status and future developments. J. Thorac. Oncol. 9(11): 1598-1608.

Chun SG, et al. 2015. Comparison of 3-D conformal and intensity modulated radiation therapy outcomes for locally advanced nonsmall cell lung cancer in NRG oncology/RTOG 0617. Int. J. Radiat. Oncol. Biol. Phys. 93(3): S1-S2.

Cosset JM, et al. 2013. Hypofractionnement en radiothérapie 1'éternel retour. Cancer Radiother: 17(5-6): 355-362.

Coutard H. 1934. Principles of X ray therapy of malignant diseases. Lancet.

Despeignes V. 1896. Observation concernant un cas de cancer de 1'estomac traité par les rayons Rontgen. Lyon Med. 82: 428.

Dieterich S, et al. 2018. SBRT targets that move with respiration. Phys. Med. 56: 19-24.

Durdux C, et al. 2018. Principes et techniques de la radiothérapie thoracique des carcinomes bronchiques non à petites cellules (CBNPC). Rev. Mal. Respir: Actual. 10(3): 303-313.

Foray N. 2016a. Les origines lyonnaises de la radiothérapie anticancéreuse. 1893-1895.

Foray N. 2016b. Victor Despeignes, the forgotten pioneer of radiation oncology. Int. J. Radiat. Oncol. Biol. Phys. 96(4): 717-721.

Franks KN, et al. 2015. Stereotactic ablative body radiotherapy for lung cancer. Clin. Oncol. 27(5): 280-289.

Hamaji M. 2019. Surgery and stereotactic body radiotherapy for early-stage non-small cell lung cancer: prospective clinical trials of the past, the present, and the future. Gen. Thorac. Cardiovasc. Surg.

Hamaji M, et al. 2019. Surgery and stereotactic body radiotherapy for early stage non-small cell lung cancer: review of meta-analyses. J. Thorac. Dis. 11(Supp1. 13): S1646-S1652.

Horn L, Johnson DH. 2008. Evarts A. Graham and the first pneumonectomy for lung cancer. J. Clin. Oncol. 26(19): 32683275.

Khaitan PG, D'Amico TA. 2018. Milestones in thoracic surgery. J. Thorac. Cardiovasc. Surg. 155(6): 2779-2789.

Koshy M, et al. 2017. Association between intensity modulated radiotherapy and survival in patients with stage III non-small cell lung cancer treated with chemoradiotherapy. Lung Cancer 108: 222-227.

Koshy M, et al. 2015. Increasing radiation therapy dose is associated with improved survival in patients undergoing stereotactic body radiation therapy for stage I non-small-cell lung cancer. Int. J. Radiat. Oncol. Biol. Phys. 91(2): 344-350.

Lanni TB, et al. 2011. Stereotactic radiotherapy reduces treatment cost while improving overall survival and local control over standard fractionated radiation therapy for medically inoperable non-small-cell lung cancer. Am. J. Clin. Oncol. Cancer Clin. Trials 34(5): 494-498.

Lefebvre L, et al. 2014. New techniques and potential benefits for radiotherapy of lung cancer. Cancer Radiother. 18(5-6): 473-479.

Liao ZX, et al. 2010. Influence of technologic advances on outcomes in patients with unresectable, locally advanced non-small-cell lung cancer receiving concomitant chemoradiotherapy. Int. J. Radiat. Oncol. Biol. Phys. 76(3): 775-781.

Menoux I, et al. 2018. Radiation-induced lung toxicity predictors after stereotactic radiation therapy for non-small cell lung carcinoma stage I. Cancer Radiother. 22(8): 826-838.

Menten MJ, et al. 2017. MRI-guided lung SBRT: present and future developments. Phys. Med. 44: 139-139.

Modlin IM, et al. 2014. A historical appreciation of bronchopulmonary neuroendocrine neoplasia. Thorac. Surg. Clin. 24(3): 235-255. 
De Neve W, et al. 1999. Modulation d'intensité en radiothérapie par collimateur multi-lame dynamique. Technique et expérience clinique. Cancer Radiother: 3(5): 378-392.

Nyman J, et al. 2016. SPACE - A randomized study of SBRT vs conventional fractionated radiotherapy in medically inoperable stage I NSCLC. Radiother. Oncol. 121(1): 1-8.

Ochsner A. 1978. The development of pulmonary surgery, with special emphasis on carcinoma and bronchiectasis. Am. J. Surg. 135(6): 732-746.

Regaud C, Blanc J. 1906. Action des rayons X sur les diverses générations de la lignée spermatique. Extrême sensibilité des spermatogonies à ces rayons. C. R. Soc. Biol.: 892 .

Shimkin MB, et al. 1962. Pneumonectomy and lobectomy in bronchogenic carcinoma. a comparison of end results of the Overholt and Ochsner clinics. J. Thorac. Cardiovasc. Surg. 44: 503-519.

Solberg JL, et al. 2012. Historical development of stereotactic ablative radiotherapy. Radiat. Oncol. 39(1): 191-197.
Videtic GM, et al. 2019. Long-term follow-up on NRG oncology RTOG 0915 (NCCTG N0927): a randomized phase 2 study comparing 2 stereotactic body radiation therapy schedules for medically inoperable patients with stage I peripheral non-small cell lung cancer. Int. J. Radiat. Oncol. Biol. Phys. 103(5): 1077-1084.

Widder J, et al. 2011. Survival and quality of life after stereotactic or 3D-conformal radiotherapy for inoperable early-stage lung cancer. Int. J. Radiat. Oncol. Biol. Phys. 81(4): 291-297.

Yaprak G, et al. 2019. Is stereotactic body radiotherapy an alternative to surgery in early stage non small cell lung cancer? J. BUON 24 (4): 1619-1625.

Yom SS, et al. 2007. Initial evaluation of treatment-related pneumonitis in advanced-stage non-small-cell lung cancer patients treated with concurrent chemotherapy and intensitymodulated radiotherapy. Int. J. Radiat. Oncol. Biol. Phys. 68(1): 94-102.
Citation de l'article : Bertho A, Dos Santos M, François A, Milliat F. 2020. Histoire de la prise en charge des cancers bronchopulmonaires non à petites cellules de stade précoce : de la chirurgie à la radiothérapie stéréotaxique. Radioprotection, https://doi.org/10.1051/radiopro/ 2020050. 


\section{Questions à l'auteur}

Q1 Merci de nous transmettre la traduction du titre en anglais.

Q2 Merci de nous transmettre la traduction des mots clés en anglais.

Q3 Merci d'ajouter les références des travaux de [Yenice et al.] et de [Shiu et al.] dans la bibliographie.

Q4 Merci de compléter les références [Coutard, 1934 et Hamaji, 2019] en nous fournissant la tomaison et les folios.

Q5 Merci de compléter la référence [Foray, 2016a] en nous fournissant le nom de la revue et la tomaison.

Q6 Merci de compléter la référence [Regaud et Blanc, 1906] en nous fournissant la tomaison. 